\title{
The Influence of Emotions and Behavioral Theories behind Hand Hygiene in COVID-19 Pandemic
}

\author{
Sheng Chuu Anne Kiew, Jia Lin Jacklyn Yek, Prit Anand Singh, Rajkumar Chandran \\ Department of Anesthesiology and Intensive Care Unit, Changi General Hospital, Singapore \\ Email: anne.kiew.s.c@singhealth.com.sg, jacklyn.yek@mohh.com.sg, singh.prit.anand@singhealth.com.sg, \\ chandran.rajkumar@singhealth.com.sg
}

How to cite this paper: Kiew, S.C.A., Yek, J.L.J., Singh, P.A. and Chandran, R. (2021) The Influence of Emotions and Behavioral Theories behind Hand Hygiene in COVID-19 Pandemic. Open Journal of Preventive Medicine, 11, 299-307. https://doi.org/10.4236/ojpm.2021.117024

Received: June 3, 2021

Accepted: July 4, 2021

Published: July 7, 2021

Copyright () 2021 by author(s) and Scientific Research Publishing Inc. This work is licensed under the Creative Commons Attribution International License (CC BY 4.0).

http://creativecommons.org/licenses/by/4.0/

(c) (i) Open Access

\begin{abstract}
Background: Hand hygiene $(\mathrm{HH})$ is essential in preventing healthcare-associated infections, especially during the COVID-19 pandemic, where SARS-CoV2 has shown the ability to survive on surfaces for days. In this study, we explore $\mathrm{HH}$ compliance rate and the factors associated with its increase during COVID-19 pandemic. Methods: HH compliance was assessed amongst doctors in Anesthesia and Surgical Intensive Care Unit by direct observation between 2018 and 2021. An anonymous survey was then sent to the doctors to understand factors influencing the observed improvement in $\mathrm{HH}$ compliance during the pandemic. Results: Compliance towards the five moments of $\mathrm{HH}$ has shown a statistically significant rising trend from a median $\mathrm{HH}$ compliance of $60 \%$ in 2018 , to $70 \%$ in 2019 to $94 \%$ in 2020 . However, HH compliance subsequently declined in the first quarter of 2021 to median of $87 \%$. The follow-up survey had a response rate of $96 \%(\mathrm{n}=53) .90 \%$ of survey participants responded that their HH frequency had increased during the COVID-19 pandemic with $47 \%$ stating they were performing HH 11 - 20 times/day. 64\% responded that this increased frequency had affected their skin condition. $62 \%$ responded that this increased frequency was sustainable even after the pandemic ends. Participants ranked considerations influencing HH compliance. "Prevent transmitting the infection to vulnerable patients" was ranked highest followed by "High infection rate", "Fear of contracting COVID-19" and lastly, "Public health guidelines". Conclusions: Despite the absence of new campaigns, $\mathrm{HH}$ compliance reached an all-time high. The pandemic has provided opportunity for behavioral change through "reflective" drivers of behavior such as emotions ("Fear") and knowledge ("High infection rate"). Understanding the motivations behind current increased $\mathrm{HH}$ compliance and riding on the increased initial uptake of behavior may help convert $\mathrm{HH}$ into a habitual action and not just a reaction to the pandemic.
\end{abstract}




\section{Keywords}

Pandemics, Hand Hygiene, Infection Control, Healthcare Associated Infection, Fear, Compliance, Behavioral Theory

\section{Introduction}

COVID-19 has spread around the world with virtually no region left untouched. The speed of the spread and the alarming death rates have seen many countries and jurisdictions introduce measures to prevent the spread of COVID-19. Handwashing has received considerable attention during the COVID-19 pandemic.

Hand hygiene (HH) is said to be the cornerstone of infection control. Lack of hand hygiene is an essential risk factor for transmitting microorganisms [1]. Microorganisms can survive on surfaces for extended periods and have often been found to contaminate and colonize Health Care Worker's (HCW) hands resulting in cross-transmission [2]. Viruses are shed in large numbers in body secretions during and after illness, including blood, feces, urine, saliva, and nasal fluid [3]. SARS-CoV and other coronaviruses can survive on environmental surfaces for up to 6 days [4]. As a result, environmental objects and surfaces become contaminated and act as fomites, while contaminated HCW hands serve as insidious vehicles for transmission. Contaminated HCW hands have been associated with endemic healthcare-associated infections (HCAI) and even outbreaks [1]. The necessity for improvement in the hand hygiene compliance rate amongst health care workers has been stressed multiple times over the years [5]. The exact contribution of hand hygiene to the reduction of the direct and indirect spread of coronaviruses between people is unknown. However, hand washing mechanically removes pathogens, and studies demonstrate that alcohol-based hand rub formulations inactivate SARS-CoV-2 [6] [7].

The results of pathogen transmission due to poor hand hygiene compliance have been extensively studied. In developed countries, $5 \%-15 \%$ of hospitalized patients have HCAIs; of which, up to $9 \%-37 \%$ require admission to intensive care units. As a result, $\mathrm{HCAI}$ is associated with prolonged hospital stay, increased resistance of microorganisms to antimicrobials, emotional stress and financial burdens for patients and their families, and increased morbidity and mortality [8].

Compliance with hand hygiene has been associated with decreased nosocomial infections. It has been shown that improved compliance with $\mathrm{HH}$ from $48 \%$ to $66 \%$, through proper training and use of alcohol hand rubs in a teaching hospital in Geneva over a span of three years, reduced nosocomial infection rates by as much as $40 \%$ [9]. Achieving high $\mathrm{HH}$ compliance rates during epidemics and pandemics is crucial to prevent the spread of infection by HCW. HH compliance rate of $100 \%$ in such times is possible, as shown by a retrospective study in China, which reported the absence of nosocomial infections during the Severe Acute 
Respiratory Syndrome outbreak [10].

HCWs across the world have responded to the pandemic with PPE measures. However, there is little detail on the HH compliance rate during the COVID-19 pandemic. Prior to the pandemic, adherence to HH amongst HCW has been reported to be variable, ranging from $5 \%$ to $89 \%$ with an overall average of $38.7 \%$ in both developed and developing countries [8]. Such a wide range of hand hygiene practice is bound to be detrimental in the COVID-19 pandemic. Compliance with hand hygiene has been unacceptably low and not sufficiently recognised by HCW [9].

The causes behind poor adherence with $\mathrm{HH}$ have been extensively studied and narrowed down to the following categories of individual factors-occupation, environmental constraints and behavioral patterns [9]. This pandemic has forced all to change their behavior and beliefs towards hand hygiene. As such, our study aims to understand the behavioral theories involved in influencing hand hygiene compliance in our hospital, which will help in promoting sustained efforts at hand washing. The focus of our study was to investigate the changes to the hand hygiene compliance rates during the pandemic, quality initiatives to improve hand hygiene compliance prior to the pandemic and study the behavior factors affecting the hand hygiene compliance in our setup.

\section{Materials and Methods}

Definition of hand hygiene referred to any action of hand cleansing. The appropriate use of alcohol-based hand rub gel or hand washing with soap and water aimed at reducing or inhibiting the growth of micro-organisms on hands is considered an essential requirement in hand hygiene. A hand hygiene opportunity was defined as a moment during health-care activities when hand hygiene is necessary to interrupt germ transmission by hands. The assessment of hand hygiene is performed by direct observation based on WHO guidelines [11]. Compliance with $\mathrm{HH}$ is the percentage of the number of performed $\mathrm{HH}$ instances to the number of $\mathrm{HH}$ opportunities.

The Hand Hygiene Observational Audit was performed by 20 nurse clinicians from the departments of Infectious Disease and Surgical Intensive Care. These nurse clinicians were adequately equipped with the skill and knowledge in $\mathrm{HH}$ and trained in assessing $\mathrm{HH}$ compliance through a half-day classroom training with simulation exercises. Detection of hand hygiene compliance by validated observers (direct observation) is currently considered the gold standard in hand hygiene compliance monitoring. Data was collated on a hard copy hand hygiene observation form and submitted to the Infection Control Department. The audit was designed based on the recommendations of World Health Organisation [11], which stated that the general guidance is that a minimum of $12-15$ observations should be in the denominator before a percentage is calculated. On selected days of a week, a minimum of 15 observations of hand hygiene opportunities (the denominator) were collected; and out of these opportunities determine the number 
of times hand hygiene was completed properly (this is the numerator) to allow computation of the percentage of hand hygiene compliance.

A sample size calculation at the design stage excludes the influence of chance. To show a difference between $70 \%$ and $95 \%$ in 2019 and 2020 respectively with a power of $90 \%$ and an alpha error of $5 \%$, two times of $44(44 \times 2)$ opportunities had to be observed. As such, a minimum of $100 \mathrm{HH}$ opportunities were conducted each week, adding up to a total of $400 \mathrm{HH}$ opportunities a month.

Convenience sampling was conducted in the form of an anonymous survey, that was sent out to the doctors of the Department of Anesthesia and Surgical Intensive Care regarding the personal views of hand hygiene and the factors influencing an increased $\mathrm{HH}$ compliance rate.

Results were analyzed using SPSS Version 25. Questions with responses that were ranked were analyzed using Friedman test.

Exemption from the review by the Singhealth Centralised Institutional Board was obtained (Ref 2020/2044).

\section{Results}

The Department of Anesthesia and Surgical Intensive Care at Changi General Hospital, a tertiary 1000-bed hospital in Singapore, achieved a high compliance rate over the period of the pandemic.

Compliance towards the five moments of $\mathrm{HH}$ has shown a statistically significant rising trend from a median $\mathrm{HH}$ compliance of $60 \%$ in 2018 , to $70 \%$ in 2019 to $94 \%$ in 2020 . However, $\mathrm{HH}$ compliance subsequently declined in first quarter of 2021 to median of $87 \%$ (Figure 1 ).

A follow up survey was conducted to obtain personal views of hand hygiene and the factors influencing an increased $\mathrm{HH}$ compliance rate amongst doctors in

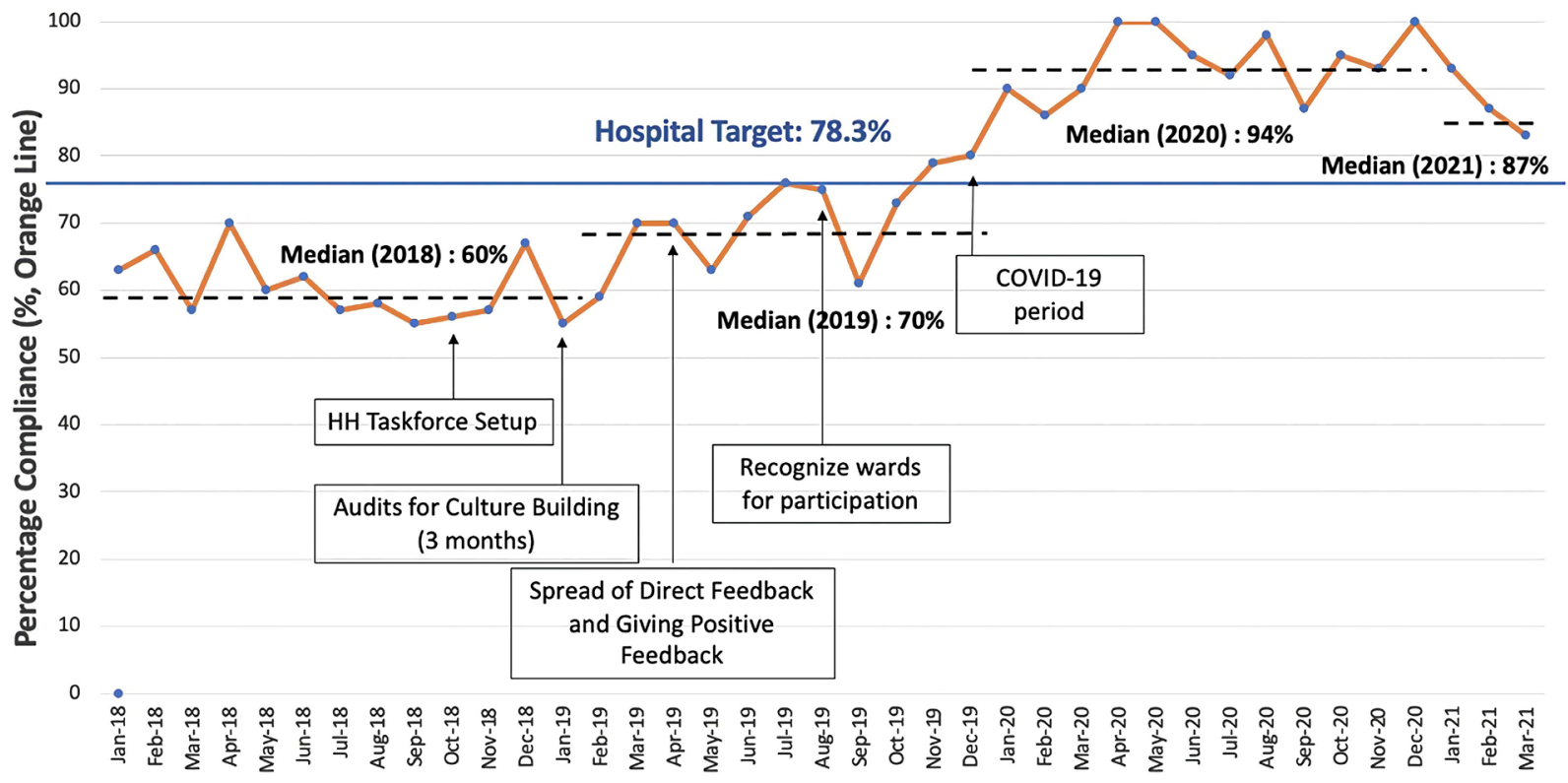

Figure 1. Hand hygiene compliance from 2018-2021 amongst anesthesia and surgical intensive care unit doctors. 
the department. The response rate of the survey was 96\% (53/55).

$96.4 \%(\mathrm{n}=53)$ responded that the frequency of hand hygiene had increased during the pandemic, with the majority $(47.2 \%, \mathrm{n}=25)$ saying $11-20$ times/day, $35.8 \%(\mathrm{n}=19)$ saying $21-50$ times/day, $13.2 \%(\mathrm{n}=7)$ saying $\leq 10$ times/day and $3.8 \%(\mathrm{n}=2)$ saying $>50$ times/day. $64.2 \%(\mathrm{n}=34)$ responded that the increased frequency had affected the skin over hands. $62.3 \%(\mathrm{n}=33)$ responded that this increased frequency is sustainable even after the end of the pandemic.

The participants were asked to rate the factors influencing them to achieve a high $\mathrm{HH}$ compliance rate on a 7-point Likert scale (1: strongly disagree to 7: strongly agree). "Preventing transmitting infection to vulnerable patients" was ranked the highest followed by "High infection rate", "Fear of contracting COVID-19" and lastly, "Public health guidelines". "Other reminders being put up e.g. posters/announcements/guidelines", "Worried about mortality rate", "Little is known about the virus e.g. mode of transmission" were ranked the lowest $(\mathrm{p}<0.001)$.

Lastly, participants were asked to rank the following three factors influencing hand hygiene from most to least important. The majority ranked fear of contracting COVID-19 as most important followed by care about patients, and lastly public health initiatives as least important. Other factors that were mentioned in the free text include less rushed workload, concern about vulnerable patients; more bottles of hand rub nearby.

\section{Discussion}

It is well known that hand hygiene is a complex behavior with a myriad of motivators and barriers. Our hospital target for $\mathrm{HH}$ compliance rate has been 78.4\% with a median of $60 \%-70 \%$ pre-COVID (Figure 1). Over the years, there have been various interventions initiated globally by the WHO such as "Clean hands save lives", "My Five Moments for Hand Hygiene" and the "Multimodal Hand Hygiene Improvement Strategy" to improve awareness about hand hygiene and generate enough motivation amongst health care workers to help decrease health care-associated infections. These have been adapted and used in our hospital with some success.

In October 2018, a surgical task force team was formed at our hospital to help further improve $\mathrm{HH}$ compliance and to raise awareness. The team was composed of doctors, nurses and allied health staff. The directives of the task force were coordinated and supported by the Office of Improvement Sciences, specially set up to assist with quality improvement initiatives in the hospital. A number of steps were carried out including hand hygiene audits along with direct feedback. Other initiatives included the recognition of wards with high participation in the direct feedback. Further hand hygiene quality initiatives by various surgical disciplines were shared with others to encourage and motivate teams to initiate quality improvement programs. HH compliance rates were mandated as a component in Junior Evaluation in an effort to further improve hand hygiene compliance. As a result of multiple initiatives in place, there was increased usage 
of alcoholic hand rubs, handwashing stations, posters, as well as high awareness and knowledge amongst HCW. Compliance rose from $56 \%$ to $73 \%$ between October 2018 and October 2019 (Figure 1).

During the COVID-19 pandemic, there have been scanty reports about $\mathrm{HH}$ compliance, despite numerous guidelines published by various health agencies. One report quoted an increase in hand hygiene rate from $54.5 \%$ to $100 \%$ in the initial months of the pandemic and only to decline to pre-pandemic rates in the subsequent months [12].

In our study, despite the absence of new HH initiatives in 2020, we witnessed a significant uptrend in compliance rates from $73 \%$ in October 2020 to $90 \%$ in March 2020 (Figure 1). We found that the main drive amongst HCW for improved $\mathrm{HH}$ was generally to prevent infection in vulnerable patients, followed by the fear of contracting COVID-19, public health guidelines and concerns of COVID-19's high infectivity. A similar phenomenon was seen during the Severe Acute Respiratory Syndrome (SARS) outbreak in 2003, where a high HH compliance (close to 90\%) amongst HCW in Singapore General Hospital was seen; and was driven primarily by fear [13]. The increased $\mathrm{HH}$ compliance rate noticed in the early months of the pandemic has shown a gradual decline but has remained higher than the pre pandemic hand hygiene compliance rates.

Many behavioral theories have been studied to understand the behavior of HCW towards hand hygiene practices. This paper attempts to discuss some of these theories in the context of the pandemic.

Firstly, the Protection Motivation Theory is likely the overarching motivation behind HH. The Protection Motivation Theory postulates that: 1) the magnitude of the noxiousness of a depicted event; 2) the probability of that event's occurrence; and 3) the efficacy of a protective response are key to a behavioral change (Rogers, 1975). The cognitive appraisal of these factors elicits motivation to protect oneself from a threat. The triggered response, as depicted by fear appeal theories, is either adaptive or maladaptive-dependent on the 1) degree of threat that is perceived, 2) response efficacy i.e. the belief that $\mathrm{HH}$ can prevent crossinfection and 3) self-efficacy i.e. the belief that one can do it. In other words, people will only respond appropriately to a health threat if they perceive themselves to be at risk and the behavior to be efficacious [14].

In the current context, the term "coronaphobia" has surfaced in studies [15]. Coronaphobia has been described as an excessive triggered response of fear of contracting the virus causing COVID-19, leading to accompanied excessive concern over physiological symptoms, significant stress about personal and occupational loss, increased reassurance and safety seeking behaviors, and avoidance of public places and situations, causing marked impairment in daily life functioning. Despite the absence of new initiatives, "reflective" drivers of behavior [13] such as knowledge ("high infectivity") and emotions ("fear") played a prominent role in hand hygiene compliance, and likely led to the phenomenal increase in the $\mathrm{HH}$ compliance rate in our hospital. 
As such intense emotions, such as fear, are not a common occurrence in the health care setup. Understanding the theoretical background of behavioral initiation is crucial in utilizing models that provide a practicable framework for designing, implementing and evaluating any intervention. These interventions will be necessary in the next challenge of converting this conscious "reflective" behavior into an unconscious "reflexive" behavior. Habits are cue-driven behaviors learnt incrementally over time through repetition, and studies suggest the use of leverage context by disruption [16] to form habitual behavior.

In the current context, disruption of usual routine by the donning of PPE (contextual cue) reminds HCW to be more mindful of circumstances and hygiene, hence allowing new opportunities for behavioral change. Psychological studies consistently show that through associative learning, mere repetition of an action in response to a consistent contextual cue results in the action being habitually performed upon subsequent exposure to those contextual cues [17]. Once initiation of the action is "transferred" to these contextual cues, dependence on conscious attention or motivational processes is reduced, resulting in habit formation.

Interestingly, although $61.5 \%$ of doctors in our department stated that this improved $\mathrm{HH}$ compliance was sustainable, the behavior was not sustained in the later part of the year. Failure of behavioral change in becoming a habit could be explained by the Theory of Rational Addiction [18] [19]. The development of habitual $\mathrm{HH}$ depends on: 1) Rationality-whether the agent conceptualises the behavior as a habit that then foresee their future consumption path given the intertemporal complementarities in behavior, and make the decision of whether or not to engage accordingly; 2) Agent's perceived returns versus true returns of an activity; and 3) Presence of social norms. Habit formation is generated by intertemporally linked preferences in consumption-the more one consumes in the past, the easier or more likely is consumption in the present. Hence, front-loading interventions to increase perceived returns and initial take up of a behavior can maximize habit stock and thereby, persistence in behavior than interventions spread over time. Additionally, interventions that monitor activity and invoke social pressure to engage can further effectively change behavior.

In the current context, hospitals can encourage habitual $\mathrm{HH}$ through incentives to maximize initial uptake and maintain the behavior through invoking social norms. This would imply directing incentives at the level of: 1) the individual (intrapersonal); 2) interactions between individuals (interpersonal); and 3) the community [20], to increase perceived benefits. Coupled with interventions that encourage $\mathrm{HH}$ as a positive social behavior, it may lead to habit formation.

Our understanding of behavioral theories and also the inability over several decades to motivate HCWs to achieve a consistently high level of compliance with $\mathrm{HH}$ suggests that changing hand hygiene behavior is a complex task [21]. Behavioral theories primarily focused on the individual have shown to be insufficient to effect sustained change. Interventions aimed at improving $\mathrm{HH}$ compliance should thus be based on the various levels of behavior interaction and 
include multiple models influencing the behavior towards hand hygiene [9].

\section{Conclusions}

Our study suggests that an increase in hand hygiene compliance can be achieved with motivation alone when all other ingredients such as education and resources are already in place. However for such results to be sustained, further interventions will be needed to face the challenge, backed with the understanding of the theories of behavioral change influencing hand hygiene behavior.

Programmes to improve hand hygiene compliance in HCWs cannot rely solely on awareness and motivational factors but must take into account the other major barriers to altering an individual's pre-existing hand hygiene behavior.

COVID-19 pandemic has resulted in an increase in $\mathrm{HH}$ adherence, which can be seen as an opportunity for behavioral change and habit formation. Institutions should capitalize on this movement as well as the social influence HCWs have on each other to help retain the improved $\mathrm{HH}$ rate as a habitual action and not just a reaction to the pandemic.

\section{Conflicts of Interest}

The authors declare no conflicts of interest regarding the publication of this paper.

\section{References}

[1] Edmonds-Wilson, S.L., Nurinova, N.I., Zapka, C.A., Fierer, N. and Wilson, M. (2015) Review of Human Hand Microbiome Research. Journal of Dermatological Science, 80, 3-12. https://doi.org/10.1016/j.jdermsci.2015.07.006

[2] Kampf, G. and Kramer, A. (2004) Epidemiologic Background of Hand Hygiene and Evaluation of the Most Important Agents for Scrubs and Rubs. Clinical Microbiology Reviews, 17, 863-893. https://doi.org/10.1128/cmr.17.4.863-893.2004

[3] Boone, S.A. and Gerba, C.P. (2007) Significance of Fomites in the Spread of Respiratory and Enteric Viral Disease. Applied and Environmental Microbiology, 73, 1687-1696. https://doi.org/10.1128/AEM.02051-06

[4] Otter, J.A., Donskey, C., Yezli, S., Douthwaite, S., Goldenberg, S.D. and Weber, D.J. (2016) Transmission of SARS and MERS Coronaviruses and Influenza Virus in Healthcare Settings: The Possible Role of Dry Surface Contamination. Journal of Hospital Infection, 92, 235-250. https://doi.org/10.1016/j.jhin.2015.08.027

[5] Erasmus, V., Kuperus, M.N., Richardus, J.H., Vos, M.C., Oenema, A. and van Beeck, E.F. (2010) Improving Hand Hygiene Behavior of Nurses Using Action Planning: A Pilot Study in the Intensive Care Unit and Surgical Ward. Journal of Hospital Infection, 76, 161-164. https://doi.org/10.1016/j.jhin.2010.04.024

[6] Centers for Disease Control and Prevention (2019) Hand Hygiene in Healthcare Settings. https://www.cdc.gov/handhygiene/index.html

[7] Kratzel, A., Todt, D., V'kovski, P., Steiner, S., Gultrom, M., Thao, T.T.N., et al. (2020) Inactivation of Severe Acute Respiratory Syndrome Coronavirus 2 by WHO-Recommended Hand Rub Formulations and Alcohols. Emerging Infectious Diseases, 26, 1592-1595. https://doi.org/10.3201/eid2607.200915

[8] World Health Organization (2009) WHO Guidelines on Hand Hygiene in Health 
Care: First Global Patient Safety Challenge Clean Care Is Safer Care. World Health Organization, Geneva.

[9] Pittet, D. (2000) Improving Compliance with Hand Hygiene in Hospitals. Infection Control \& Hospital Epidemiology, 21, 381-386. https://doi.org/10.1086/501777

[10] World Health Organization (2009) WHO Guidelines on Hand Hygiene in Health Care: First Global Patient Safety Challenge Clean Care Is Safer Care-Hand Hygiene as a Performance Indicator. World Health Organization, Geneva. https://www.ncbi.nlm.nih.gov/books/NBK144028/

[11] Jiang, H.-P., Zhou, B.-P., Lu, P.-X. and Yu, W.-Y. (2004) Preventive Effects against SARS among Medical Personnel in Hospital. Chinese Journal of Nosoconmiology, 14, 906-908.

[12] Makhni, S., Umscheid, C.A., Soo, J., Chu, V., Bartlett, A., Landon, E. and Marrs, R. (2021) Hand Hygiene Compliance Rate during the COVID-19 Pandemic. JAMA Internal Medicine, Article No. e211429. https://doi.org/10.1001/jamainternmed.2021.1429

[13] Ling, M.L. and How, K.B. (2012) Impact of a Hospital-Wide Hand Hygiene Promotion Strategy on Healthcare-Associated Infections. Antimicrobial Resistance and Infection, 1, Article No. 13. https://doi.org/10.1186/2047-2994-1-13

[14] Jenner, E.A., Jones, F., Fletcher, B.C., Miller, L. and Scott, G.M. (2005) Hand Hygiene Posters: Selling the Message. Journal of Hospital Infection, 59, 77-82. https://doi.org/10.1016/j.jhin.2004.07.002

[15] Arora, A., Jha, A.K., Alat, P. and Das, S.S. (2020) Understanding Coronaphobia. Asian Journal of Psychiatry, 54, Article ID: 102384. https://doi.org/10.1016/j.ajp.2020.102384

[16] Neal, D., Vujcic, J., Hernandez, O. and Wood, W. (2015) The Science of Habit: Creating Disruptive and Sticky Behavior Change in Handwashing Behavior. Washington DC, USA.

https://globalhandwashing.org/wp-content/uploads/2019/03/the-science-of-habit-cr eating-disruptive-and-sticky-behavior-change-in-handwashing-behavior.pdf

[17] Gardner, B., Lally, P. and Wardle, J. (2012) Making Health Habitual: The Psychology of 'Habit-Formation' and General Practice. British Journal of General Practice, 62, 664-666. https://doi.org/10.3399/bjgp12x659466

[18] Becker, G. and Murphy, K. (1988) A Theory of Rational Addiction. Journal of Political Economy, 96, 675-700. http://www.jstor.org/stable/1830469

[19] Reshmaan Nahar, H., Atonu, R., Giovanni, R. and Natalia, R. (2017) Habit Formation and Rational Addiction: A Field Experiment in Handwashing. Harvard Business School, Working Paper No. 18-030.

[20] Pittet, D. (2004) The Lowbury Lecture: Behaviour in Infection Control. Journal of Hospital Infection, 58, 1-13. https://doi.org/10.1016/j.jhin.2004.06.002

[21] Larson, E. and Kretzer, E.K. (1995) Compliance with Handwashing and Barrier Precautions. Journal of Hospital Infection, 30, 88-106. https://doi.org/10.1016/0195-6701(95)90010-1 